\title{
Direction-cosine-matrix-based attitude control subject to actuator saturation
}

ISSN 1751-8644

Received on 26th November 2013

Accepted on 6th February 2015

doi: 10.1049/iet-cta.2014.0890

www.ietdl.org

\section{James Richard Forbes ${ }^{凶}$ \\ Department of Aerospace Engineering, University of Michigan, FXB Building, 320 Beal Avenue, Ann Arbor, Michigan 48109-2140, USA $\bowtie$ E-mail:forbesrj@umich.edu}

\begin{abstract}
Set-point attitude control of a rigid body explicitly preventing actuator saturation is considered. The attitude control approach developed does not employ any sort of direction-cosine-matrix (DCM) parameterisation, such as Euler angles or quaternions. Rather, the DCM is used directly within the feedback control algorithm. Together a proportional control term and an angular velocity control term make up the attitude controller. The angular velocity control is composed of a strictly positive real system subject to a special input non-linearity. The specific form of the proportional control and angular velocity control ensure control torques are below the saturation level of the on-board actuators. Two controller synthesis methods are considered. The first uses the linearised system, the solution to the linear quadratic regulator problem, and the Kalman-Yakubovich-Popov lemma to design the controller. The second employs a simple low-pass filter that is guaranteed to stabilise the closed-loop system; tuning the low-pass filter is also considered. Numerical simulation results demonstrate effective closed-loop control in the presence of plant disturbances and sensor noise.
\end{abstract}

\section{Introduction}

Robotic systems that are free to rotate in space require a robust attitude control system to realise their mission objectives. For instance, spacecraft must slew and point in order to observe celestial objects, and for inspection purposes underwater vehicles must manoeuvre safely around newly constructed or ageing dams, bridge abutments and other marine structures.

The set of attitudes a rigid body may take is the set of $3 \times 3$ orthonormal matrices with determinant equal to plus one. The direction-cosine-matrix (DCM) or the rotation matrix describes the attitude of a rigid body globally and uniquely, unlike parameterisations of the DCM, such as Euler angles, Rodrigues parameters, modified Rodrigues parameters (MRPs) or unit-length quaternions (referred to as quaternions for simplicity). Many research articles have considered the use of DCM parameterisations for control of rigid bodies [1-6]. Unfortunately, all DCM parameterisations are deficient in some way. For instance, Euler angles, Rodrigues parameters and MRPs suffer from singularities, while the same physical attitude is represented by two (antipodal) quaternions [7]. In fact, the use of quaternions for attitude control can lead to undesirable rotations known as unwinding [7, 8]. As such, researchers and practitioners alike are presently focusing on attitude control methods that use the DCM directly [7, 9-14]. Given the modern advances in on-board computing, DCM-based estimators and controllers can be implemented on-board flight vehicles without much difficulty $[15,14-18]$.

From an applications point of view it is ideal when practical limitations, such as actuator saturation and noise rejection are accounted for directly in the control formulation, rather than patched with ad-hoc fixes during implementation. The articles [19-22] consider attitude control in the presence of actuator saturation, however, attitude is parameterised using quaternions. In $[19,20]$ hyperbolic tangent functions are employed to prevent actuator saturation, whereas saturation functions are used in $[21,22]$. Attitude control in the presence of saturation is also considered in $[10,11]$ where the DCM is used directly for control. To the knowledge of the author, the existing literature, including [10, 11, $19,19,21,22]$, has yet to consider closed-loop attitude control using the DCM directly while simultaneously employing dynamic angular velocity control and disallowing actuator saturation. Doing so is the motivation and focus of this paper.

This paper considers the design of an attitude control law that does not use a DCM parameterisation, but rather uses the DCM directly for attitude control. Specifically, set-point attitude control is considered where the attitude controller is composed of a proportional control component and an angular velocity control component. The proportional control component is borrowed from [13], and is a function of the DCM describing the attitude error. The angular velocity control component is inspired from [23, 24], and is composed of a strictly positive real (SPR) system with a specific input non-linearity. It is shown that together the control torque produced by the proportional and angular velocity controls can be limited, thereby avoiding actuator (magnitude) saturation. Moreover, the control architecture guarantees a desired equilibrium point of the closed-loop system is asymptotically stable. The novelty of this work lies in the ability to prevent actuator saturation while guaranteeing robust closed-loop asymptotic stability of a desired equilibrium point using the attitude error DCM and an SPR system directly within the control law. The introduction of the SPR system enables the rejection of noise in the angular velocity measurement. Two straightforward SPR design methods are considered. The first uses the linearised system along with [25] to synthesise an SPR controller. The second method considers the design of a filtered proportional derivative (PD) control law, a control scheme often used in practice over a standard PD control law in order to filter noise corrupting the angular velocity measurement. Tuning of the filtered PD controller using a steady-state error analysis of the closed-loop system is considered representing another modest contribution.

The remainder of this paper is as follows. In Section 2, preliminaries are reviewed, such as the kinematics and dynamics of a rigid body. In Section 3, attitude error and various functions related to attitude error used to prove stability will be discussed. The control structure is presented in Section 4, and the means by which actuator saturation is prevented is demonstrated. Closed-loop asymptotic stability of a desired equilibrium is proven in Section 5. Controller synthesis is considered in Section 6. Numerical simulation results are presented in Section 7. The paper is drawn to a close in Section 8. 


\section{Preliminaries}

Consider a rigid body, such as a spacecraft or underwater vehicle. Let $\mathcal{F}_{a}$ and $\mathcal{F}_{b}$ denote the inertial and body frames, where the origin of the body frame is located at the mass centre of the rigid body. The attitude of $\mathcal{F}_{b}$ relative $\mathcal{F}_{a}$ is globally and uniquely described by the DCM $\mathbf{C}_{b a} \in S O(3)$, where $S O(3)=$ $\left\{\mathbf{C} \in \mathbb{R}^{3 \times 3} \mid \mathbf{C}^{\top} \mathbf{C}=\mathbf{1}, \operatorname{det} \mathbf{C}=+1\right\}$ is the special orthogonal group of rigid-body rotations $[26,27]$. Some authors prefer to work with the rotation matrix which is the transpose of $\mathbf{C}_{b a}$. The control method presented herein can be adopted to work with the rotation matrix. The kinematics and dynamics governing the motion of the rigid body are given by the Poisson and Euler equations [26, 28]

$$
\begin{aligned}
\dot{\mathbf{C}}_{b a}+\omega^{\times} \mathbf{C}_{b a} & =\mathbf{0} \\
\mathbf{I} \dot{\boldsymbol{\omega}}+\boldsymbol{\omega}^{\times} \mathbf{I} \boldsymbol{\omega} & =\boldsymbol{\tau}_{\mathrm{c}}
\end{aligned}
$$

where $\omega$ is the angular velocity of $\mathcal{F}_{b}$ with respect to $\mathcal{F}_{a}, \mathbf{I}=\mathbf{I}^{\top}>$ 0 is the moment of inertia of the rigid body and $\boldsymbol{\tau}_{\mathrm{c}}$ is the control torque. All quantities in (2) are expressed in $\mathcal{F}_{b}$.

The 'cross' map $(.)^{\times}: \mathbb{R}^{3} \rightarrow \mathfrak{s o}(3)$ transforms a $3 \times 1$ column matrix to a $3 \times 3$ skewsymmetric matrix

$$
\mathbf{v}^{\times}=\left[\begin{array}{ccc}
0 & -v_{3} & v_{2} \\
v_{3} & 0 & -v_{1} \\
-v_{2} & v_{1} & 0
\end{array}\right], \quad \forall \mathbf{v}=\left[\begin{array}{l}
v_{1} \\
v_{2} \\
v_{3}
\end{array}\right] \in \mathbb{R}^{3}
$$

where the vector space of all $3 \times 3$ skewsymmetric matrices is $\mathfrak{s o}(3)=\left\{\mathbf{S} \in \mathbb{R}^{3 \times 3} \mid \mathbf{S}^{\top}=-\mathbf{S}\right\}[26,27]$. The 'uncross' map (.) $)^{\mathrm{v}}:$ $\mathfrak{s o}(3) \rightarrow \mathbb{R}^{3}$ does just the opposite, transforming a $3 \times 3$ skewsymmetric matrix into $3 \times 1$ column matrix so that $\mathbf{v}^{\times \mathbf{v}}=\mathbf{v}, \forall \mathbf{v} \in \mathbb{R}^{3}$. A useful identity that employs both $(\cdot)^{\times}$and $(\cdot)^{\mathrm{V}}$ is

$$
\frac{1}{2} \operatorname{tr}\left(\mathbf{v}^{\times} \mathbf{U}\right)=-\mathbf{v}^{\top} \mathcal{P}_{a}(\mathbf{U})^{\mathbf{v}}, \quad \forall \mathbf{v} \in \mathbb{R}^{3}, \quad \forall \mathbf{U} \in \mathbb{R}^{3 \times 3}
$$

where $\mathcal{P}_{a}(\mathbf{U})=\frac{1}{2}\left(\mathbf{U}-\mathbf{U}^{\top}\right)$ is the skewsymmetric projection operator.

DCM parameterisations, such as Euler axis/angle parameters and quaternions, are often used to describe attitude. In this paper, DCM parameterisations will not be used within the control scheme developed, however, axis/angle parameters and quaternions will be referred to from time-to-time. Let $(\mathbf{a}, \phi)$ be the Euler axis and Euler angle, respectively, where $\mathbf{a} \in \mathbb{S}^{2}, \phi \in \mathbb{R}$ and $\mathbb{S}^{2}$ is the two-sphere. Let $\mathbf{q}=\left[\begin{array}{ll}\boldsymbol{\epsilon}^{\top} & \eta\end{array}\right]^{\top} \in \mathbb{S}^{3}$ denote the (unit-length) quaternion, where $\epsilon \in \mathbb{R}^{3}$ is the vector part of the quaternion, $\eta \in \mathbb{R}$ is the scalar part of the quaternion and $\mathbb{S}^{3}$ is the three-sphere. The vector and scalar parts of the quaternion can be written in terms of a and $\phi$ in the following ways: $\epsilon=\sin (\phi / 2)$ a and $\eta=\cos (\phi / 2)$. An arbitrary DCM, $\mathbf{C} \in S O(3)$, can be written in terms of either of these parameterisations [28]

$$
\begin{aligned}
& \mathbf{C}(\mathbf{a}, \phi)=\cos \phi \mathbf{1}+(1-\cos \phi) \mathbf{a a}^{\top}-\sin \phi \mathbf{a}^{\times}=e^{-\phi \mathbf{a}^{\times}} \\
& \mathbf{C}(\boldsymbol{\epsilon}, \eta)=\left(\eta^{2}-\boldsymbol{\epsilon}^{\top} \boldsymbol{\epsilon}\right) \mathbf{1}+2 \boldsymbol{\epsilon} \boldsymbol{\epsilon}^{\top}-2 \eta \boldsymbol{\epsilon}^{\times}
\end{aligned}
$$

In the upcoming proof of closed-loop asymptotic stability, the following lemma will be employed.

Lemma 1 Kalman-Yakubovich-Popov (KYP) lemma [29]: Consider the LTI system

$$
\dot{\mathbf{x}}_{\mathrm{c}}=\mathbf{A}_{\mathrm{c}} \mathbf{x}_{\mathrm{c}}+\mathbf{B}_{\mathrm{c}} \mathbf{u}_{\mathrm{c}}, \quad \mathbf{y}_{\mathrm{c}}=\mathbf{C}_{\mathrm{c}} \mathbf{x}_{\mathrm{c}}
$$

where $\mathbf{x}_{\mathrm{c}} \in \mathbb{R}^{n_{\mathrm{c}}}, \mathbf{u}_{\mathrm{c}}$ and $\mathbf{y}_{\mathrm{c}} \in \mathbb{R}^{m}$ are the matrices $\mathbf{A}_{\mathrm{c}}, \mathbf{B}_{\mathrm{c}}$ and $\mathbf{C}_{\mathrm{c}}$ are appropriately dimensioned real matrices that form a minimal statespace realisation, and $\mathbf{A}_{\mathrm{c}}$ is Hurwitz. The system is SPR if and only if there exists $\mathbf{P}_{\mathrm{c}} \in \mathbb{R}^{n_{\mathrm{c}} \times n_{\mathrm{c}}}$ and $\mathbf{Q}_{\mathrm{c}} \in \mathbb{R}^{n_{\mathrm{c}} \times n_{\mathrm{c}}}$ where $\mathbf{P}_{\mathrm{c}}=\mathbf{P}_{\mathrm{c}}^{\top}>0$ and $\mathbf{Q}_{\mathrm{c}}=\mathbf{Q}_{\mathrm{c}}^{\top}>0$ such that

$$
\begin{aligned}
\mathbf{P}_{\mathrm{c}} \mathbf{A}_{\mathrm{c}}+\mathbf{A}_{c}^{\top} \mathbf{P}_{\mathrm{c}} & =-\mathbf{Q}_{\mathrm{c}} \\
\mathbf{P}_{\mathrm{c}} \mathbf{B}_{\mathrm{c}} & =\mathbf{C}_{c}^{\top}
\end{aligned}
$$

\section{Attitude error}

This paper will focus on regulation of a rigid body's attitude to a constant desired attitude. Let $\mathbf{C}_{\mathrm{da}}$ be the DCM describing the desired attitude. The attitude error described by the attitude error $\mathrm{DCM}$ is $\mathbf{E}=\mathbf{C}_{b d}=\mathbf{C}_{b a} \mathbf{C}_{\mathrm{da}}^{\top}$. The attitude error kinematics are

$$
\dot{\mathbf{E}}+\omega^{\times} \mathbf{E}=\mathbf{0}
$$

Equation (6) comes from differentiating $\mathbf{E}$ with respect to time and using (1)

Consider the following attitude error function [13]

$$
\psi(\mathbf{E})=2-\sqrt{1+\operatorname{tr}(\mathbf{E})}
$$

This attitude error function will be used for stability analysis via Lyapunov's direct method in Section 5. Let $\left(\mathbf{a}_{e}, \phi_{e}\right)$ be the Euler axis/angle associated with $\mathbf{E}$. Recall that $-1 \leq \operatorname{tr}(\mathbf{E}) \leq 3$, where $\operatorname{tr}(\mathbf{E})=3$ only when $\mathbf{E}=\mathbf{1}$ (i.e. when $\phi_{e}=0$ ), and $\operatorname{tr}(\mathbf{E})=-1$ only when $\mathbf{E}=e^{ \pm \pi \mathbf{a}_{e}}, \forall \mathbf{a}_{e} \in \mathbb{S}^{2}$ [28]. Thus, $\psi(\mathbf{E})=0$ if and only if $\mathbf{E}=\mathbf{1}$, and $\psi(\mathbf{E})=2$ if and only if $\mathbf{E}=e^{ \pm \pi \mathbf{a}_{e}}, \forall \mathbf{a}_{e} \in \mathbb{S}^{2}$. Define the sublevel set $\mathfrak{L}_{2}=\{\mathbf{E} \in S O(3) \mid \psi(\mathbf{E})<2\}$. Notice that attitudes corresponding to $\mathbf{E}=e^{ \pm \pi \mathbf{a}_{e}}, \forall \mathbf{a}_{e} \in \mathbb{S}^{2}$ are disallowed in the sublevel set $\mathfrak{L}_{2}$.

Consider the following attitude error column matrix $[13,30]$

$$
\mathbf{e}=\frac{1}{\sqrt{1+\operatorname{tr}(\mathbf{E})}} \mathcal{P}_{a}(\mathbf{E})^{\vee}
$$

where $\mathbf{e}$ is well defined in the sublevel set $\mathfrak{L}_{2}$. In terms of Euler axis/angle parameters $\left(\mathbf{a}_{e}, \phi_{e}\right)$ the attitude error column matrix $\mathbf{e}$ is

$$
\begin{aligned}
\mathbf{e} & =\frac{1}{\sqrt{1+\operatorname{tr}(\mathbf{E})}} \mathcal{P}_{a}(\mathbf{E})^{\mathrm{v}} \\
& =\frac{1}{\sqrt{1+\left(3 \cos \phi_{e}+\left(1-\cos \phi_{e}\right) \mathbf{a}_{e}^{\top} \mathbf{a}_{e}\right)}}\left(-\sin \phi_{e} \mathbf{a}_{e}^{\times}\right)^{\mathrm{v}} \\
& =-\frac{1}{\sqrt{2\left(1+\cos \phi_{e}\right)}} \sin \phi_{e} \mathbf{a}_{e} \\
& =-\frac{1}{\sqrt{\cos ^{2} \frac{\phi_{e}}{2}}} \sin \frac{\phi_{e}}{2} \cos \frac{\phi_{e}}{2} \mathbf{a}_{e} \\
& =-\sin \frac{\phi_{e}}{2} \mathbf{a}_{e} \operatorname{sign}\left(\cos \frac{\phi_{e}}{2}\right)
\end{aligned}
$$

where $\mathbf{a}_{e}^{\top} \mathbf{a}_{e}=1, x / \sqrt{x^{2}}=\operatorname{sign}(x) \forall x \neq 0$ where $\operatorname{sign}(x)=+1$ if $x>0$ and $\operatorname{sign}(x)=-1$ if $x<0$, and the identities $\cos ^{2}\left(\phi_{e} / 2\right)=$ $\left(1+\cos \phi_{e}\right) / 2$ and $\sin \phi_{e}=2 \sin \left(\phi_{e} / 2\right) \cos \left(\phi_{e} / 2\right)$ have been used to simplify. As such, in the sublevel set $\mathfrak{L}_{2}, \mathbf{e}=\mathbf{0}$ if and only if $\phi_{e}=0$, and hence $\mathbf{E}=\mathbf{1}$. Also, let $\left(\boldsymbol{\epsilon}_{e}, \eta_{e}\right)$ where $\boldsymbol{\epsilon}_{e}=$ $\left[\begin{array}{lll}\epsilon_{e, 1} & \epsilon_{e, 2} & \epsilon_{e, 3}\end{array}\right]^{\top}$ be the error quaternion associated with $\mathbf{E}$. Given the relationship between $\mathbf{a}_{e}, \phi_{e}$ and $\boldsymbol{\epsilon}_{e}$ [i.e. $\boldsymbol{\epsilon}_{e}=\sin \left(\phi_{e} / 2\right) \mathbf{a}_{e}$ and $\left.\eta_{e}=\cos \left(\phi_{e} / 2\right)\right]$, it follows that $\mathbf{e}=-\boldsymbol{\epsilon}_{e} \operatorname{sign}\left(\eta_{e}\right)$. Thus, in the sublevel set $\mathfrak{L}_{2},\left|e_{i}\right| \leq 1, i=1,2,3$, where $\mathbf{e}=\left[\begin{array}{lll}e_{1} & e_{2} & e_{3}\end{array}\right]^{\top}$, which follows from $\left|\epsilon_{e, i}\right| \leq 1, i=1,2,3$. The fact that the absolute value of each element of $\mathbf{e}$ is bounded by one will be exploited when designing a controller that does not saturate on-board actuators.

The attitude error function in (7) and associated attitude error column matrix in (8) have several advantages over others found in the literature. As discussed in [13], the primary advantage is that the Euclidean norm of $\mathbf{e},\|\mathbf{e}\|$, approaches one as $\phi_{e}$ approaches $180^{\circ}$. The norm of other attitude error column matrices found in the literature approach zero as $\phi_{e}$ approaches $180^{\circ}$, which is undesirable because it degrades closed-loop performance when used within a feedback control strategy. See [13] for details. 


\section{Control formulation}

Owing to hardware limitations the control torque that can be applied is limited. To avoid actuator saturation the control torque, $\boldsymbol{\tau}_{\mathrm{c}}=\left[\begin{array}{lll}\tau_{\mathrm{c}, 1} & \tau_{\mathrm{c}, 2} & \tau_{\mathrm{c}, 3}\end{array}\right]^{\top}$, will be designed so that the magnitude of the total torque in each axis, $\left|\tau_{\mathrm{c}, i}\right|, i=1,2,3$, is less than the maximum torque the on-board actuators can apply, $0<\bar{\tau}_{\mathrm{c}, i}<\infty$, $i=1,2,3$.

To this end, consider the following control structure

$$
\begin{aligned}
\boldsymbol{\tau}_{\mathrm{c}} & =\boldsymbol{\tau}_{p}+\boldsymbol{\tau}_{d} \\
& =k \mathbf{e}-\boldsymbol{\sigma}(\mathbf{u})
\end{aligned}
$$

The proportional control is $\boldsymbol{\tau}_{p}=k \mathbf{e}$ where $0<k<\infty$ is the proportional control gain. The angular velocity control is $\tau_{d}=-\sigma(\mathbf{u})$ where $\sigma: \mathbb{R}^{3} \rightarrow \mathbb{R}^{3}$ is the plant input non-linearity, $\mathbf{u}=\mathbf{y}_{\mathrm{c}}$ is the output of the system

$$
\begin{aligned}
\dot{\mathbf{x}}_{\mathrm{c}} & =\mathbf{A}_{\mathrm{c}} \mathbf{x}_{\mathrm{c}}+\mathbf{B}_{\mathrm{c}} \mathbf{u}_{\mathrm{c}}=\mathbf{A}_{\mathrm{c}} \mathbf{x}_{\mathrm{c}}+\mathbf{B}_{\mathrm{c}} \hat{\mathbf{y}} \\
\mathbf{u}=\mathbf{y}_{\mathrm{c}} & =\mathbf{C}_{\mathrm{c}} \mathbf{x}_{\mathrm{c}}
\end{aligned}
$$

where the transfer matrix $\mathbf{G}_{\mathrm{c}}(s)=\mathbf{C}_{\mathrm{c}}\left(s \mathbf{1}-\mathbf{A}_{\mathrm{c}}\right)^{-1} \mathbf{B}_{\mathrm{c}}$ is SPR. The input to $\mathbf{G}_{\mathrm{c}}(s)$ is $\mathbf{u}_{\mathrm{c}}=\hat{\mathbf{y}}=\boldsymbol{\beta}(\mathbf{u}) \boldsymbol{\omega}$ where $\boldsymbol{\beta}: \mathbb{R}^{3} \rightarrow \mathbb{R}^{3 \times 3}$ is the controller input non-linearity. A block diagram of the closed-loop system is shown in Fig. 1. The proportional control, the nonlinearities $\sigma(\cdot)$ and $\boldsymbol{\beta}(\cdot)$, and the SPR system are all specifically chosen in order to guarantee that actuator saturation is prevented and asymptotic stability of a desired equilibrium point of the closed-loop system is achieved.

Before considering the specifics of the non-linearities $\sigma(\cdot)$ and $\boldsymbol{\beta}(\cdot)$, and the SPR system that generates $\mathbf{u}=\left[\begin{array}{lll}u_{1} & u_{2} & u_{3}\end{array}\right]^{\top}$, the means by which actuator saturation is prevented will be discussed. First, let $\boldsymbol{\tau}_{p}=\left[\begin{array}{lll}\tau_{p, 1} & \tau_{p, 2} & \tau_{p, 3}\end{array}\right]^{\top}$ and $\boldsymbol{\tau}_{d}=\left[\begin{array}{lll}\tau_{d, 1} & \tau_{d, 2} & \tau_{d, 3}\end{array}\right]^{\top}$. Recall from Section 3 that $\left|e_{i}\right| \leq 1, i=1,2,3$. Therefore each element of the proportional control is bounded by $k$, that is $\left|\tau_{p, i}\right|=$ $k\left|e_{i}\right| \leq k, i=1,2,3$. It follows that provided $k+\bar{u}_{i}<\bar{\tau}_{\mathrm{c}, i}$ where $\left|\tau_{d, i}\right| \leq \bar{u}_{i}$ and $0<\bar{u}_{i}<\infty$ is the maximum allowable angular velocity control in the $i$ th axis (where $i=1,2,3$ ), then the control torque in each axis will be less than the maximum allowable torque in each axis, $\bar{\tau}_{\mathrm{c}, i}$, thereby preventing actuator saturation. Note that $k$ and $\bar{u}_{i}$ can be chosen so that their sum is arbitrary close to $\bar{\tau}_{\mathrm{c}, i}$.

The details of $\sigma(\cdot)$ and $\beta(\cdot)$ will now be discussed. The nonlinearity $\sigma(\cdot)$ has the following specific form $[23,24]$

$$
\begin{aligned}
& \boldsymbol{\sigma}(\mathbf{u})=\left[\begin{array}{lll}
\sigma_{1}\left(u_{1}\right) & \sigma_{2}\left(u_{2}\right) & \sigma_{3}\left(u_{3}\right)
\end{array}\right]^{\top} \\
& \sigma_{i}\left(u_{i}\right)= \begin{cases}\bar{u}_{i} & \bar{u}_{i}<u_{i} \\
u_{i} & -\bar{u}_{i} \leq u_{i} \leq \bar{u}_{i}, \quad i=1,2,3 \\
-\bar{u}_{i} & u_{i}<-\bar{u}_{i}\end{cases}
\end{aligned}
$$

where as previously mentioned, $0<\bar{u}_{i}<\infty$ is the maximum allowable angular velocity control in the $i$ th axis. Notice that each

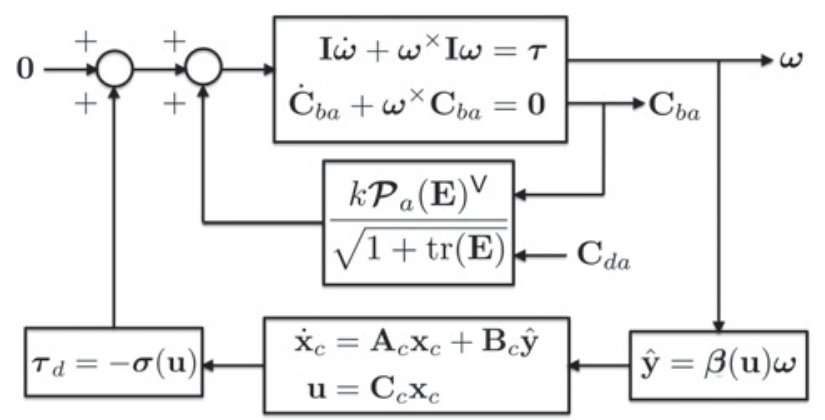

Fig. 1 Block diagram of closed-loop system $\bar{u}_{i}$ can be different, thus realising different saturation levels in each axis. Following $[23,24]$, the function $\boldsymbol{\beta}(\cdot)$ is defined as

$$
\beta(\mathbf{u}) \mathbf{u}=\sigma(\mathbf{u})
$$

where $\boldsymbol{\beta}(\mathbf{u})=\operatorname{diag}\left\{\beta_{1}\left(u_{1}\right), \beta_{2}\left(u_{2}\right), \beta_{3}\left(u_{3}\right)\right\}=\boldsymbol{\beta}^{\top}(\mathbf{u})$ and

$$
\beta_{i}\left(u_{i}\right)=\left\{\begin{array}{ll}
\sigma_{i}\left(u_{i}\right) / u_{i} & u_{i} \neq 0 \\
1 & u_{i}=0
\end{array}, \quad i=1,2,3\right.
$$

As the $\bar{u}_{i}$ are user-defined parameters and each $u_{i}$ is known at all times during operation, both $\sigma(\cdot)$ and $\beta(\cdot)$ can be computed online and in real time. Observe that when $-\bar{u}_{i} \leq u_{i} \leq \bar{u}_{i}$ that $\beta_{i}\left(u_{i}\right)=1$, and when $\bar{u}_{i}<u_{i}$ or $u_{i}<-\bar{u}_{i}$ that $\beta_{i}\left(u_{i}\right)<1$. As such, $\beta_{i}(\cdot)$ strictly less than one indicates the angular velocity control is being limited, thereby preventing saturation. Using (11) notice that

$$
\omega^{\top} \boldsymbol{\sigma}(\mathbf{u})=\omega^{\top} \boldsymbol{\beta}(\mathbf{u}) \mathbf{u}=(\boldsymbol{\beta}(\mathbf{u}) \boldsymbol{\omega})^{\top} \mathbf{u}=\hat{\mathbf{y}}^{\top} \mathbf{u}, \quad \forall \boldsymbol{\omega}, \mathbf{u} \in \mathbb{R}^{3}
$$

where $\omega$ could be replaced with an arbitrary column matrix. This inner product relationship is deliberate, and will be exploited when assessing closed-loop asymptotic stability of a descried equilibrium point.

Prior to showing that a desired equilibrium point of the closedloop system is in fact asymptotically stable, a conservative aspect of the control scheme will be discussed. Consider the case where, at an instant, $e_{i}<1$ in the $i$ th axis, and thus $\tau_{p, i}<k$. At the same instant, consider when the angular velocity control in the same $i$ th axis is such that $\tau_{d, i}=\bar{u}_{i}$. In this case, for saturation to be avoided in the $i$ th axis, the angular velocity control does not have to be restricted by an amount $\bar{u}_{i}$, and could in fact take on a larger value. The proposed control architecture is conservative in that the angular velocity control is restricted without knowledge or consideration of the concurrent proportional control component. Doing so ensures that actuator saturation is avoided, but may also degrade closedloop performance. However, although conservative, robust closedloop stability can be guaranteed, as shown in Section 5. Therefore, as with many control engineering challenges, there is a the tradeoff between performance and a closed-loop stability guarantee.

\section{$5 \quad$ Closed-loop stability analysis}

Consider the closed-loop system collectively described by (2), (6) and (9)-(11). It can be shown that $\left(\mathbf{E}, \boldsymbol{\omega}, \mathbf{x}_{\mathrm{c}}\right)=(\mathbf{1}, \mathbf{0}, \mathbf{0})$ is an equilibrium point of the closed-loop system. Asymptotic stability of this equilibrium point will now be considered.

Theorem 1: Consider a rigid body described by the dynamics of (2). The attitude error kinematics are given by (6) where $\mathbf{E}=$ $\mathbf{C}_{b d}=\mathbf{C}_{b a} \mathbf{C}_{\mathrm{da}}^{\top}$ and $\mathbf{C}_{\mathrm{da}}$ is the DCM associated with the desired and constant attitude. The control law is given by (9) where $\mathbf{u}=\mathbf{y}_{\mathrm{c}}$, $\mathbf{y}_{\mathrm{c}}(s)=\mathbf{G}_{\mathrm{c}}(s) \mathbf{u}_{\mathrm{c}}(s), \mathbf{u}_{\mathrm{c}}=\hat{\mathbf{y}}=\boldsymbol{\beta}(\mathbf{u}) \boldsymbol{\omega}, \mathbf{G}_{\mathrm{c}}(s)=\mathbf{C}_{\mathrm{c}}\left(s \mathbf{1}-\mathbf{A}_{\mathrm{c}}\right)^{-1} \mathbf{B}_{\mathrm{c}}$, where $\left(\mathbf{A}_{\mathrm{c}}, \mathbf{B}_{\mathrm{c}}, \mathbf{C}_{\mathrm{c}}\right)$ is a minimal state-space realisation satisfying the KYP lemma, $\mathbf{A}_{\mathrm{c}}$ is Hurwitz, and $\mathbf{B}_{\mathrm{c}}$ has full column rank. If the initial conditions are such that

$$
\frac{1}{2} \omega^{\top}(0) \mathbf{I} \boldsymbol{\omega}(0)+k(2-\sqrt{1+\operatorname{tr}(\mathbf{E}(0))})+\frac{1}{2} \mathbf{x}_{\mathrm{c}}^{\top}(0) \mathbf{P}_{\mathrm{c}} \mathbf{x}_{\mathrm{c}}(0)<2 k
$$

then $\left(\mathbf{E}, \boldsymbol{\omega}, \mathbf{x}_{\mathrm{c}}\right) \rightarrow(\mathbf{1}, \mathbf{0}, \mathbf{0})$ as $t \rightarrow \infty$ and the equilibrium point $\left(\mathbf{E}, \boldsymbol{\omega}, \mathbf{x}_{\mathrm{c}}\right)=(\mathbf{1}, \mathbf{0}, \mathbf{0})$ is asymptotically stable.

Proof: Consider the following positive definite Lyapunov function candidate

$$
V=\frac{1}{2} \omega^{\top} \mathbf{I} \boldsymbol{\omega}+k(2-\sqrt{1+\operatorname{tr}(\mathbf{E})})+\frac{1}{2} \mathbf{x}_{\mathrm{c}}^{\top} \mathbf{P}_{\mathrm{c}} \mathbf{x}_{\mathrm{c}}
$$


Taking the derivative and simplifying using (2), (3), (6), (9)-(11) and the KYP lemma gives

$$
\begin{aligned}
\dot{V}= & \omega^{\top}\left(-\omega^{\times} \mathbf{I} \boldsymbol{\omega}+\boldsymbol{\tau}_{\mathrm{c}}\right)-\frac{k \operatorname{tr}(\dot{\mathbf{E}})}{2 \sqrt{1+\operatorname{tr}(\mathbf{E})}} \\
& +\frac{1}{2} \mathbf{x}_{\mathrm{c}}^{\top}\left(\mathbf{P}_{\mathrm{c}} \mathbf{A}_{\mathrm{c}}+\mathbf{A}_{\mathrm{c}}^{\top} \mathbf{P}_{\mathrm{c}}\right) \mathbf{x}_{\mathrm{c}}+\mathbf{x}_{\mathrm{c}}^{\top} \mathbf{P}_{\mathrm{c}} \mathbf{B}_{\mathrm{c}} \hat{\mathbf{y}} \\
= & \omega^{\top}\left(\frac{k \mathcal{P}_{a}(\mathbf{E})^{\mathrm{v}}}{\sqrt{1+\operatorname{tr}(\mathbf{E})}}-\sigma(\mathbf{u})\right)+\frac{k \operatorname{tr}\left(\boldsymbol{\omega}^{\times} \mathbf{E}\right)}{2 \sqrt{1+\operatorname{tr}(\mathbf{E})}} \\
& -\frac{1}{2} \mathbf{x}_{\mathrm{c}}^{\top} \mathbf{Q}_{\mathrm{c}} \mathbf{x}_{\mathrm{c}}+\mathbf{x}_{\mathrm{c}}^{\top} \mathbf{C}_{\mathrm{c}}^{\top} \hat{\mathbf{y}} \\
= & -\omega^{\top} \boldsymbol{\sigma}(\mathbf{u})+\frac{k \omega^{\top} \mathcal{P}_{a}(\mathbf{E})^{\mathrm{v}}}{\sqrt{1+\operatorname{tr}(\mathbf{E})}}-\frac{k \omega^{\top} \mathcal{P}_{a}(\mathbf{E})^{\mathrm{v}}}{\sqrt{1+\operatorname{tr}(\mathbf{E})}}-\frac{1}{2} \mathbf{x}_{\mathrm{c}}^{\top} \mathbf{Q}_{\mathrm{c}} \mathbf{x}_{\mathrm{c}}+\mathbf{u}^{\top} \hat{\mathbf{y}} \\
= & -\omega^{\top} \boldsymbol{\beta}(\mathbf{u}) \mathbf{u}-\frac{1}{2} \mathbf{x}_{\mathrm{c}}^{\top} \mathbf{Q}_{\mathrm{c}} \mathbf{x}_{\mathrm{c}}+\mathbf{u}^{\top} \boldsymbol{\beta}(\mathbf{u}) \boldsymbol{\omega} \\
= & -\frac{1}{2} \mathbf{x}_{\mathrm{c}}^{\top} \mathbf{Q}_{\mathrm{c}} \mathbf{x}_{\mathrm{c}}
\end{aligned}
$$

which implies $V(t) \leq V(0)$ for all $t \geq 0$. Combining this result with (12) it can be seen that $k \psi(\mathbf{E}(t)) \leq V(t) \leq V(0)<2 k$. Therefore, the sublevel set $\mathfrak{L}_{2}$ is a positively invariant set, and as such the attitude error column matrix $\mathbf{e}$ is well defined. 'En route' to showing asymptotic stability consider the following. First, let $\Omega=\left\{(\mathbf{E}, \omega, \mathbf{x}) \in S O(3) \times \mathbb{R}^{3} \times \mathbb{R}^{n_{\mathrm{c}}} \mid V(t) \leq V(0)\right\}$ and $\mathcal{E}=\{(\mathbf{E}, \boldsymbol{\omega}, \mathbf{x}) \in \Omega \mid \dot{V}=0\}$. Owing to the fact that $\mathbf{Q}_{\mathrm{c}}=\mathbf{Q}_{\mathrm{c}}^{\top}>0$, $\dot{V}=0$ only if $\mathbf{x}_{\mathrm{c}}=\mathbf{0}$, which in turn implies $\dot{\mathbf{x}}_{\mathrm{c}}=\mathbf{0}$ and $\mathbf{u}=\mathbf{0}$ (= $\mathbf{y}_{\mathrm{c}}$ ). With $\mathbf{x}_{\mathrm{c}}=\dot{\mathbf{x}}_{\mathrm{c}}=\mathbf{0}$, it follows that $\mathbf{B}_{\mathrm{c}} \hat{\mathbf{y}}=\mathbf{0}$, and because $\mathbf{B}_{\mathrm{c}}$ has full column rank this implies $\hat{\mathbf{y}}=\mathbf{0}$. This in turn implies $\boldsymbol{\omega}=\mathbf{0}$ via $\hat{\mathbf{y}}=\boldsymbol{\beta}(\mathbf{u}) \boldsymbol{\omega}$ where $\beta_{i}\left(u_{i}\right) \neq 0, \forall u_{i} \in \mathbb{R}, i=1,2$, 3. From $\boldsymbol{\omega}=\mathbf{0}$, we have $\dot{\boldsymbol{\omega}}=\mathbf{0}$. In addition, from (2), (9) and the positive invariance of the sublevel set $\mathfrak{L}_{2}$ we have that $\mathbf{e}=\mathbf{0}$. Following the discussion in Section 3, $\mathbf{e}=\mathbf{0}$ means $\mathbf{E}=\mathbf{1}$. Therefore the largest invariant set in $\mathcal{E}$ is the set $\left.\mathcal{M}=\left\{\left(\mathbf{E}, \boldsymbol{\omega}, \mathbf{x}_{\mathrm{c}}\right) \in \mathcal{E} \mid \mathbf{E}=\mathbf{1}, \boldsymbol{\omega}=\mathbf{0}, \mathbf{x}_{\mathrm{c}}=\mathbf{0}\right)\right\}$. From LaSalle's invariance principle it follows that trajectories of the closed-loop system asymptotically approach $\mathcal{M}$, that is, $\left(\mathbf{E}, \boldsymbol{\omega}, \mathbf{x}_{\mathrm{c}}\right) \rightarrow(\mathbf{1}, \mathbf{0}, \mathbf{0})$ as $t \rightarrow \infty$.

Of note is the fact that the proof of stability does not depend on precise knowledge of the inertia matrix. As such, our control scheme is robust to such modelling errors.

The constraint on the initial conditions given in (12) is needed to ensure that the sublevel set $\mathfrak{L}_{2}$ is a positively invariant set, and hence $\mathbf{e}$ is well defined. Similar initial condition constraints can be found in $[9,12,13]$. For an initial attitude error, angular velocity, controller states, $k$, and $\mathbf{P}_{\mathrm{c}}$, if (12) is not satisfied then one may pick a $k$ that is larger (as well as a different $\mathbf{P}_{\mathrm{c}}$, if so desired) in order to satisfy (12). If $k$ changes a different set of $\bar{u}_{i}, i=1,2,3$, parameters will most likely have to be used as well in order to ensure actuator saturation is prevented. These changes will also change the closed-loop system response.

\section{Strictly positive real controller synthesis}

\subsection{Six-state controller}

The state-space matrices of the SPR system given in (10) will first be designed using the linear quadratic regulator (LQR) formulation and the KYP lemma, as in [25]. To begin, the plant including the proportional control is linearised

$$
\begin{aligned}
{\left[\begin{array}{l}
\dot{\theta} \\
\ddot{\theta}
\end{array}\right] } & =\underbrace{\left[\begin{array}{cc}
\mathbf{0} & \mathbf{1} \\
-\mathbf{I}^{-1} \mathbf{K} & \mathbf{0}
\end{array}\right]}_{\mathbf{A}} \underbrace{\left[\begin{array}{c}
\boldsymbol{\theta} \\
\dot{\theta}
\end{array}\right]}_{\mathbf{X}}+\underbrace{\left[\begin{array}{c}
\mathbf{0} \\
\mathbf{I}^{-1}
\end{array}\right]}_{\mathbf{B}} \sigma(\mathbf{u}) \\
\mathbf{y} & =\underbrace{\left[\begin{array}{ll}
\mathbf{0} & \mathbf{1}
\end{array}\right]}_{\mathbf{C}}\left[\begin{array}{c}
\boldsymbol{\theta} \\
\dot{\theta}
\end{array}\right]
\end{aligned}
$$

where $\mathbf{e} \doteq-(1 / 2) \boldsymbol{\theta}, \boldsymbol{\omega} \doteq \dot{\boldsymbol{\theta}}$ and $\dot{\boldsymbol{\omega}} \doteq \ddot{\boldsymbol{\theta}}$, which follows from $\mathbf{E} \doteq$ $\mathbf{1}-\boldsymbol{\theta}^{\times}$where $\boldsymbol{\theta}$ are small angles involving three principal axes, and hence $\mathbf{K}=(k / 2) \mathbf{1}$. Next, using $\mathbf{A}$ and $\mathbf{B}$ from (15a), along with the user-defined weights $\mathbf{Q}_{\mathrm{LQR}}$ and $\mathbf{R}_{\mathrm{LQR}}$, the state-feedback gain $\mathbf{C}_{\mathrm{c}}=\mathbf{R}_{\mathrm{LQR}}{ }^{-1} \mathbf{B}^{\top} \boldsymbol{\Pi}$ is found by solving the algebraic Riccati equation, $\boldsymbol{\Pi} \mathbf{A}+\mathbf{A}^{\top} \boldsymbol{\Pi}-\boldsymbol{\Pi} \mathbf{B} \mathbf{R}_{\mathrm{LOR}}^{-1} \mathbf{B}^{\top} \boldsymbol{\Pi}+\mathbf{Q}_{\mathrm{LQR}}=\mathbf{0}$, for $\boldsymbol{\Pi}$. Setting $\mathbf{A}_{\mathrm{c}}=\mathbf{A}-\mathbf{B C}_{\mathrm{c}}$ and using (5b) of the KYP lemma, $\mathbf{B}_{\mathrm{c}}=$ $\mathbf{P}_{\mathrm{c}}^{-1} \mathbf{C}_{\mathrm{c}}^{\top}$ is found where $\mathbf{P}_{\mathrm{c}}$ is the solution to (5a) given the weight $\mathbf{Q}_{\mathrm{c}}$. The resultant system is SPR. This SPR controller has six states, which is why it is called the six-state controller. Note that the linearisation procedure employed above is done strictly to design the six-state controller. The six-state controller, which is SPR, is used within (9) to control the non-linear system described by (2) and (6), not a linearised version of (2) and (6)

\subsection{Filtered PD controller}

Next, a very simple SPR controller will be considered. To motivate the form of the controller, consider a PD controller of the form

$$
\boldsymbol{\tau}_{\mathrm{c}}=k \mathbf{e}-\sigma\left(k_{d} \omega\right)
$$

where $0<k<\infty$ is the proportional control gain and $0<k_{d}<\infty$ is the derivative control gain. Using a similar Lyapunov function to the one shown in (13) (just with the $(1 / 2) \mathbf{x}_{\mathrm{c}}^{\top} \mathbf{P}_{\mathrm{c}} \mathbf{x}_{\mathrm{c}}$ term removed), it can be shown that this control law renders the desired equilibrium point of the closed-loop system asymptotically stable. In addition, provided that $k+\bar{u}_{i}<\bar{\tau}_{\mathrm{c}, i}, i=1,2,3$, saturation is avoided. Unfortunately, high-frequency noise in the angular velocity measurement can be amplified. Consider the case where the state-space matrices of (10) are

$$
\begin{aligned}
& \mathbf{A}_{\mathrm{c}}=\operatorname{diag}\{-1 / T,-1 / T,-1 / T\}, \quad \mathbf{B}_{\mathrm{c}}=1 / T \mathbf{1}, \\
& \mathbf{C}_{\mathrm{c}}=k_{d} \mathbf{1}
\end{aligned}
$$

where $0<T<\infty$. The angular velocity control in each axis is effectively $\tau_{d, i}=-\sigma_{i}\left(u_{i}\right), u_{i}(s)=g_{\mathrm{c}}(s) \hat{y}_{i}(s)$ where $g_{\mathrm{c}}(s)=$ $k_{d} /(T s+1)$. It is straightforward to verify that $g_{\mathrm{c}}(s)$ is SPR. Using the $\mathbf{A}_{\mathrm{c}}, \mathbf{B}_{\mathrm{c}}$ and $\mathbf{C}_{\mathrm{c}}$ given in (17) within (9) and (10) effectively realises a filtered $\mathrm{PD}$ controller where the angular velocity measurement is passed through a low-pass filter and actuator saturation is guaranteed to be prevented.

How to pick $T$ given the presence of noise in both the attitude and angular velocity measurements will now be discussed. For comparison purposes, the closed-loop performance at steady state when using the PD controller in (16) will be compared to the closed-loop performance at steady state when using (9) and (10) where $\mathbf{A}_{\mathrm{c}}, \mathbf{B}_{\mathrm{c}}$ and $\mathbf{C}_{\mathrm{c}}$ are given in (17). Consider the case where the closed-loop system has almost reached the desired equilibrium point of $\mathbf{E}=\mathbf{1}$ and $\boldsymbol{\omega}=\mathbf{0}$ so that both $\mathbf{e}$ and $\omega$ are small and there is no actuator saturation. For small angles and rates $\mathbf{e} \doteq-(1 / 2) \boldsymbol{\theta}$, $\boldsymbol{\omega} \doteq \dot{\boldsymbol{\theta}}, \dot{\boldsymbol{\omega}} \doteq \ddot{\boldsymbol{\theta}}$ and the $i$ th component of the PD controller in (16) is

$$
\tau_{\mathrm{c}, i}=-\frac{k}{2}\left(\theta_{i}+v_{e, i}\right)-k_{d}\left(\dot{\theta}_{i}+v_{\omega, i}\right)=\mathbf{K}_{\mathrm{PD}} \mathbf{x}_{\mathrm{PD}, i}+\mathbf{K}_{\mathrm{PD}} \mathbf{v}_{i}
$$

where

$$
\mathbf{x}_{\mathrm{PD}, i}=\left[\begin{array}{ll}
\theta_{i} & \dot{\theta}_{i}
\end{array}\right]^{\top}, \quad \mathbf{v}_{i}=\left[\begin{array}{ll}
v_{e, i} & v_{\omega, i}
\end{array}\right]^{\top}, \quad \mathbf{K}_{\mathrm{PD}}=\left[\begin{array}{ll}
-\frac{k}{2} & -k_{d}
\end{array}\right]
$$

and $\mathbf{v}_{i}$ is zero mean Gaussian noise with $E\left[\mathbf{v}_{i} \mathbf{v}_{i}^{\top}\right]=\mathbf{R}=$ $\operatorname{diag}\left\{r_{e}, r_{\omega}\right\}>0$ and $E\left[\mathbf{v}_{i} \mathbf{x}_{\mathrm{PD}, i}^{\top}\right]=\mathbf{0}$. Assuming $\mathbf{I}=\operatorname{diag}\left\{I_{1}, I_{2}, I_{3}\right\}$ the closed-loop dynamics in the $i$ th axis of the linearised system controlled using (16) are

$$
\left[\begin{array}{c}
\dot{\theta}_{i} \\
\ddot{\theta}_{i}
\end{array}\right]=\underbrace{\left[\begin{array}{cc}
0 & 1 \\
-\frac{k}{2 I_{i}} & -\frac{k_{d}}{I_{i}}
\end{array}\right]}_{\mathbf{A}_{\mathrm{PD}, i}}\left[\begin{array}{c}
\theta_{i} \\
\dot{\theta}_{i}
\end{array}\right]+\underbrace{\left[\begin{array}{cc}
0 & 0 \\
-\frac{k}{2 I_{i}} & -\frac{k_{d}}{I_{i}}
\end{array}\right]}_{\boldsymbol{\Gamma}_{\mathrm{PD}, i}}\left[\begin{array}{c}
v_{e, i} \\
v_{\omega, i}
\end{array}\right]
$$

Let $\mathbf{P}_{\mathrm{PD}, i}=E\left[\mathbf{x}_{\mathrm{PD}, i} \mathbf{x}_{\mathrm{PD}, i}^{\top}\right]$ be the state covariance. Denoting the steady-state covariance as $\mathbf{P}_{\mathrm{PD}, i}^{\mathrm{Ss}}=\lim _{t \rightarrow \infty} \mathbf{P}_{\mathrm{PD}, i}$, the steady-state 
covariance satisfies

$$
\mathbf{A}_{\mathrm{PD}, i} \mathbf{P}_{\mathrm{PD}, i}^{\mathrm{sS}}+\mathbf{P}_{\mathrm{PD}, i}^{\mathrm{ss}} \mathbf{A}_{\mathrm{PD}, i}^{\top}+\boldsymbol{\Gamma}_{\mathrm{PD}, i} \mathbf{R} \Gamma_{\mathrm{PD}, i}^{\top}=\mathbf{0}
$$

Let $\mathbf{P}_{\mathrm{PD}, i}^{\mathrm{ss}}$ be parameterised as

$$
\mathbf{P}_{\mathrm{PD}, i}^{\mathrm{ss}}=\left[\begin{array}{ll}
p_{1, i} & p_{\triangle, i} \\
p_{\triangle, i} & p_{2, i}
\end{array}\right]
$$

Using (19), the elements of $\mathbf{P}_{\mathrm{PD}, i}^{\mathrm{ss}}, p_{1, i}, p_{\triangle, i}$ and $p_{2, i}$, can be found

$$
\begin{aligned}
p_{1, i} & =\frac{k}{4 k_{d}} r_{e}+\frac{k_{d}}{k} r_{\omega} \\
p_{\triangle, i} & =0 \\
p_{2, i} & =\frac{k^{2}}{8 k_{d} I_{i}} r_{e}+\frac{k_{d}}{2 I_{i}} r_{\omega}
\end{aligned}
$$

The steady-state control covariance about the $i$ th axis, $p_{\mathrm{PD}, i}^{\tau}=E\left[\tau_{\mathrm{c}, i}^{2}\right]$ where $\tau_{\mathrm{c}, i}$ is given in (18), can also be computed:

$$
\begin{aligned}
p_{\mathrm{PD}, i}^{\tau} & =\mathbf{K}_{\mathrm{PD}}\left(\mathbf{P}_{\mathrm{PD}, i}^{\mathrm{Ss}}+\mathbf{R}\right) \mathbf{K}_{\mathrm{PD}}^{\top} \\
& =\left(\frac{k^{3}}{16 k_{d}}+\frac{k^{2} k_{d}}{8 I_{i}}+\frac{k^{2}}{4}\right) r_{e}+\left(\frac{k_{d}^{3}}{2 I_{i}}+k_{d}^{2}+\frac{k k_{d}}{4}\right) r_{\omega}
\end{aligned}
$$

Next, consider the closed-loop system controlled using the SPR controller given by (9) and (10) where $\mathbf{A}_{\mathfrak{c}}, \mathbf{B}_{\mathrm{c}}$ and $\mathbf{C}_{\mathrm{c}}$ are given in (17). Again, assume that the closed-loop system is close to the desired equilibrium point so that $\mathbf{e} \doteq-(1 / 2) \boldsymbol{\theta}, \omega \doteq \dot{\boldsymbol{\theta}}$ and $\dot{\boldsymbol{\omega}} \doteq \ddot{\boldsymbol{\theta}}$. The $i$ th component of the control is

$$
\begin{aligned}
\tau_{\mathrm{c}, i} & =-\frac{k}{2}\left(\theta_{i}+v_{e, i}\right)-k_{d} x_{c, i} \\
\dot{x}_{\mathrm{c}, i} & =-\frac{1}{T} x_{\mathrm{c}, i}+\frac{1}{T} \dot{\theta}_{i}+\frac{1}{T} v_{\omega, i}
\end{aligned}
$$

The closed-loop dynamics in the $i$ th axis of the linearised system are

$$
\left[\begin{array}{c}
\dot{\theta}_{i} \\
\ddot{\theta}_{i} \\
\dot{x}_{\mathrm{c}, i}
\end{array}\right]=\underbrace{\left[\begin{array}{ccc}
0 & 1 & 0 \\
-\frac{k}{2 I_{i}} & 0 & -\frac{k_{d}}{I_{i}} \\
0 & \frac{1}{T} & -\frac{1}{T}
\end{array}\right]}_{\mathbf{A}_{\mathrm{SPR}, i}} \underbrace{\left[\begin{array}{c}
\theta_{i} \\
\dot{\theta}_{i} \\
x_{c, i}
\end{array}\right]}_{\mathbf{x}_{\mathrm{SPR}, i}}+\underbrace{\left[\begin{array}{cc}
0 & 0 \\
-\frac{k}{2 I_{i}} & 0 \\
0 & \frac{1}{T}
\end{array}\right]}_{\boldsymbol{\Gamma}_{\mathrm{SPR}, i}} \mathbf{v}_{i}
$$

Letting $\mathbf{P}_{\mathrm{SPR}, i}=E\left[\mathbf{x}_{\mathrm{SPR}, i} \mathbf{x}_{\mathrm{SPR}, i}^{\top}\right]$ be the state covariance, the steady-state covariance $\mathbf{P}_{\mathrm{SPR}, i}^{\mathrm{SS}}=\lim _{t \rightarrow \infty} \mathbf{P}_{\mathrm{SPR}, i}$ satisfies

$$
\mathbf{A}_{\mathrm{SPR}, i} \mathbf{P}_{\mathrm{SPR}, i}^{\mathrm{SS}}+\mathbf{P}_{\mathrm{SPR}, i}^{\mathrm{SS}} \mathbf{A}_{\mathrm{SPR}, i}^{\top}+\boldsymbol{\Gamma}_{\mathrm{SPR}, i} \mathbf{R} \Gamma_{\mathrm{SPR}, i}^{\top}=\mathbf{0}
$$

Let $\mathbf{P}_{\mathrm{SPR}, i}^{\mathrm{SS}}$ be written as

$$
\mathbf{P}_{\mathrm{SPR}, i}^{\mathrm{SS}}=\left[\begin{array}{lll}
p_{11, i} & p_{12, i} & p_{13, i} \\
p_{12, i} & p_{22, i} & p_{23, i} \\
p_{13, i} & p_{23, i} & p_{33, i}
\end{array}\right]
$$

From (24), the elements of $\mathbf{P}_{\mathrm{SPR}, i}^{\mathrm{Ss}}$ can be solved for

$$
p_{11, i}=p_{1, i}+\frac{T^{2} k^{2}}{8 k_{d} I_{i}} r_{e}
$$

$$
\begin{aligned}
& p_{12, i}=0 \\
& p_{13, i}=\frac{T^{2}}{k^{2}} 8 k_{d} I_{i} r_{e} \\
& p_{22, i}=p_{2, i}+\left(\frac{T^{2} k^{3}}{16 k_{d} I_{i}^{2}}+\frac{T k^{2}}{8 I_{i}^{2}}\right) r_{e} \\
& p_{23, i}=\frac{k^{2}}{8 k_{d} I_{i}} r_{e} \\
& p_{33, i}=\frac{k^{2}}{8 k_{d} I_{i}} r_{e}+\frac{1}{2 T} r_{\omega}
\end{aligned}
$$

where $p_{1, i}$ and $p_{i, 2}$ are given in (20) and (21). The steady-state control covariance about the $i$ th axis is

$$
\begin{aligned}
p_{\mathrm{SPR}, i}^{\tau} & =E\left[\tau_{\mathrm{c}, i}^{2}\right] \\
& =\mathbf{K}_{\mathrm{SPR}} \mathbf{P}_{\mathrm{SPR}, i}^{\mathrm{SS}} \mathbf{K}_{\mathrm{SPR}}^{\top}+\mathbf{K}_{\mathrm{SPR}, v} \mathbf{R K}_{\mathrm{SPR}, v}{ }^{\top} \\
& =p_{\mathrm{PD}, i}^{\tau}+\frac{T k^{3}}{8 I_{i}}\left(\frac{T k}{4 k_{d}}+1\right) r_{e}+\left(k_{d}^{2}\left(\frac{1}{2 T}-1\right)-\frac{k_{d}^{3}}{2 T}\right) r_{\omega}
\end{aligned}
$$

where $\tau_{\mathrm{c}, i}$ is given in (23)

$$
\mathbf{K}_{\mathrm{SPR}}=\left[\begin{array}{lll}
-\frac{k}{2} & 0 & -k_{d}
\end{array}\right] \text { and } \mathbf{K}_{\mathrm{SPR}, v}=\left[\begin{array}{ll}
-\frac{k}{2} & 0
\end{array}\right]
$$

Comparing (20), (21), (25) and (26) it can be seen that $p_{11, i}>p_{1, i}$ and $p_{22, i}>p_{2, i}$ for any $0<T<\infty$. However, by appropriate choice of $T$ (e.g. $0<T<1)$ the difference between $p_{11, i}$ and $p_{1, i}$ along with $p_{22, i}$ and $p_{2, i}$ can be mitigated. Also, from (22) and (28), by appropriately choosing $T, p_{\mathrm{PD}, i}^{\tau}>p_{\mathrm{SPR}, i}^{\tau}$, meaning that the steady-state control covariance of the SPR controller will be strictly less than that of the PD controller. This result is somewhat expected; high-frequency noise in the angular velocity measurement is filtered out thereby preventing high-frequency oscillations in the control signal, which in turn reduces the steady-state control covariance.

\section{Simulation results}

Three simulations will be presented, each considering the attitude control of a rigid-body spacecraft. The following parameters are common to all simulations. The spacecraft is in a circular orbit at an inclination of $60^{\circ}$ and altitude of $550(\mathrm{~km})$. The angle of the right ascension of the ascending node is set to zero. The inertia matrix of the spacecraft is $\mathbf{I}=\operatorname{diag}\{15,10,17.5\}\left(\mathrm{kg} \cdot \mathrm{m}^{2}\right)$. The control torques are realised by a set of reaction wheels that are limited to torques of $\bar{\tau}_{\mathrm{c}, i}=0.01 \mathrm{~N} \cdot \mathrm{m}$ in each axis. The desired attitude is $\mathbf{C}_{\mathrm{da}}=\mathbf{C}_{1}\left(5^{\circ}\right) \mathbf{C}_{2}\left(15^{\circ}\right) \mathbf{C}_{3}\left(-45^{\circ}\right)$, where $\mathbf{C}_{1}, \mathbf{C}_{2}$ and $\mathbf{C}_{3}$ are principal DCMs about the 1,2 and 3 axes. Specifically, $\mathbf{C}_{1}(\alpha)$ is computed by setting $\mathbf{a}=\left[\begin{array}{lll}1 & 0 & 0\end{array}\right]^{\top}$ and $\phi=\alpha$ in (4), $\mathbf{C}_{2}(\beta)$ is computed by setting $\mathbf{a}=\left[\begin{array}{lll}0 & 1 & 0\end{array}\right]^{\top}$ and $\phi=\beta$ in (4), and $\mathbf{C}_{3}(\gamma)$ is computed by setting $\mathbf{a}=\left[\begin{array}{lll}0 & 0 & 1\end{array}\right]^{\top}$ and $\phi=\gamma$ in (4) [28]. Two disturbance torques are considered. The first is a gravity gradient disturbance torque, $\boldsymbol{\tau}_{\text {gg }}=\left(3 \mu / r^{5}\right) \mathbf{r}^{\times} \mathbf{I r}$, where $\mu$ is the Earth's gravitational constant, $\mathbf{r}$ is the spacecraft position (expressed in the spacecraft body frame) and $r=$ $\sqrt{\mathbf{r}^{\top} \mathbf{r}}$ [28]. The second is a residual magnetic disturbance torque, $\boldsymbol{\tau}_{m}=\mathbf{m}_{r}^{\times} \mathbf{b}$, where $\mathbf{m}_{r}=\left[\begin{array}{lll}0.1 & 0.1 & 0.1\end{array}\right]^{\top}\left(\mathrm{A} \cdot \mathrm{m}^{2}\right)$ is the residual magnetic dipole and $\mathbf{b} \in \mathbb{R}^{3}$ is the Earth's magnetic field vector expressed in the spacecraft body frame [28]. The angular velocity is corrupted by noise of the form $\mathbf{v}_{\omega}=(0.0005 \sin (250 t)+$ $0.0001 \sin (500 t+\pi / 4))\left[\begin{array}{lll}1 & 1 & 1\end{array}\right]^{\top}(\mathrm{rad} / \mathrm{s})$. The attitude error DCM is constructed (estimated) from noisy vector measurements using the TRIAD algorithm [31] in the following way. Let $\mathbf{y}_{a}^{1}=\left[\begin{array}{lll}1 & 0 & 0\end{array}\right]^{\top}$ and $\mathbf{y}_{a}^{2}=\left[\begin{array}{lll}0 & 1 & 0\end{array}\right]^{\top}$ be reference vectors expressed in the inertial frame (frame $\mathcal{F}_{a}$ ). The reference vectors expressed in the desired frame (frame $\mathcal{F}_{d}$ ) 

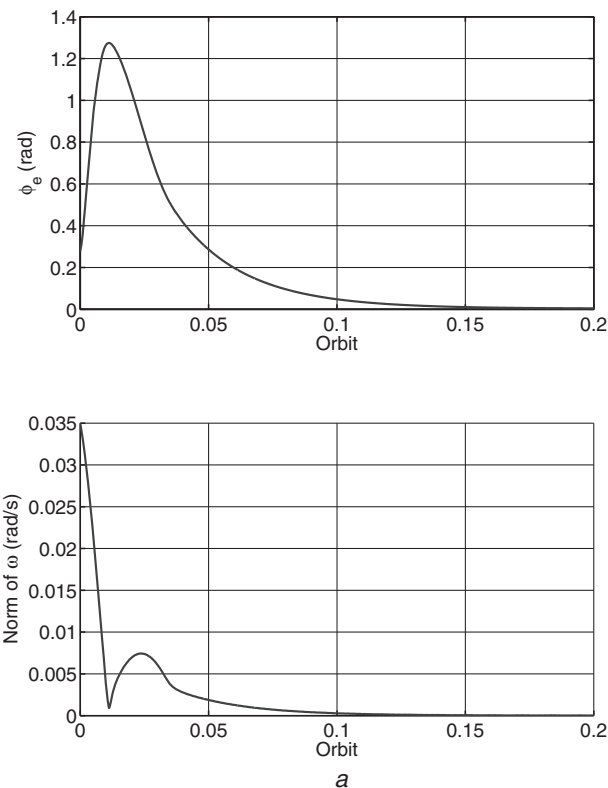
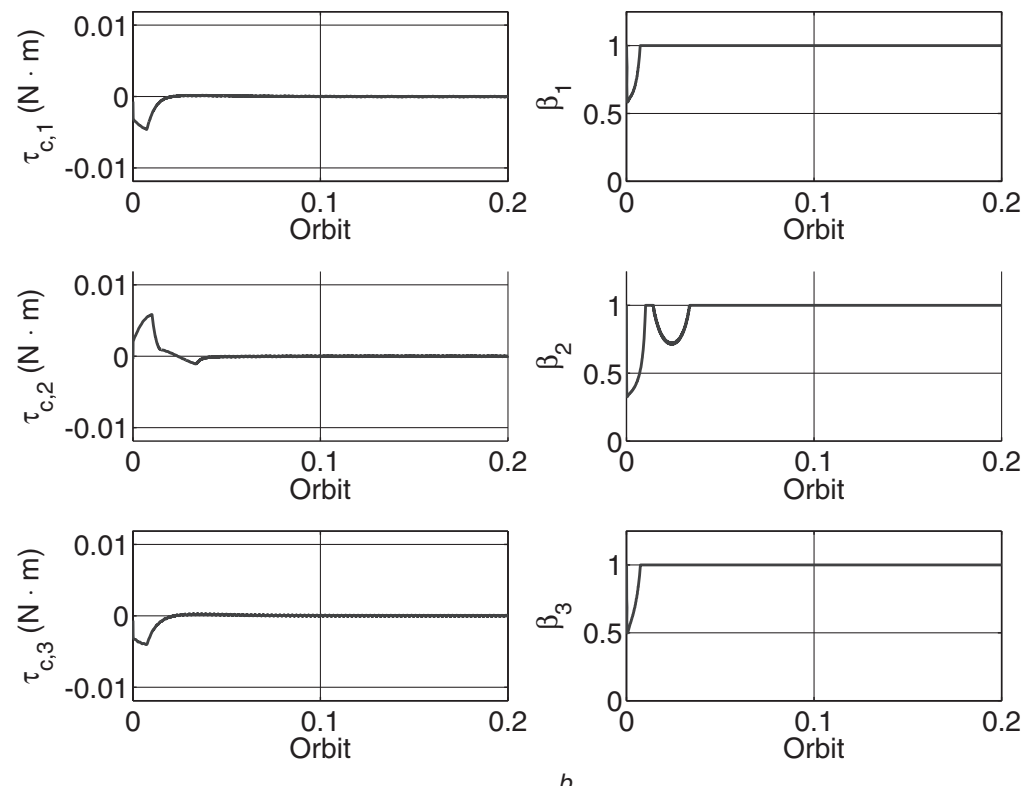

Fig. 2 Closed-loop system response; perfect measurements and large initial attitude error

$a$ Attitude error against orbit and norm of angular velocity against orbit

$b$ Control torque against orbit and $\beta_{i}(\cdot)$ against orbit

are $\mathbf{y}_{d}^{j}=\mathbf{C}_{\mathrm{da}} \mathbf{y}_{a}^{j}, j=1,2$. The vector measurements are generated as follows: $\mathbf{y}_{b}^{j}=\mathbf{C}_{v b}^{j} \mathbf{C}_{b a} \mathbf{y}_{a}^{j}, j=1,2$, where $\mathbf{C}_{v b}^{j}$ are noise DCMs. Specifically, $\mathbf{C}_{v b}^{1}=\mathbf{C}_{3}\left(v_{3,1} \sin \left(v_{p, 1} t\right)\right) \mathbf{C}_{2}\left(v_{2,1} \cos \left(v_{p, 1} t\right)\right)$ $\mathbf{C}_{1}\left(v_{1,1} \sin \left(v_{p, 1} t\right)\right)$ where $v_{p, 1}=1 / 2 \mathrm{~s}^{-1}, v_{1,1}=1 / 4^{\circ}, v_{2,1}=1 / 2^{\circ}$, $v_{3,1}=1^{\circ}$ and $\mathbf{C}_{v b}^{2}=\mathbf{C}_{3}\left(v_{3,2} \cos \left(v_{p, 2} t\right)\right) \mathbf{C}_{2}\left(v_{2,2} \sin \left(v_{p, 2} t\right)\right) \mathbf{C}_{1}\left(v_{1,2}\right.$ $\left.\cos \left(v_{p, 2} t\right)\right)$ where $v_{p, 2}=1 / 10 \mathrm{~s}^{-1}, \quad v_{1,2}=3 / 10^{\circ}, \quad v_{2,2}=1 / 5^{\circ}$, $v_{3,2}=1 / 10^{\circ}$. Using the TRIAD algorithm, the attitude error $\mathrm{DCM}$ is $\mathbf{E}=\left[\begin{array}{llll}\mathbf{v}_{b}^{1} & \mathbf{v}_{b}^{2} & \mathbf{v}_{b}^{3}\end{array}\right]\left[\begin{array}{lll}\mathbf{v}_{d}^{1} & \mathbf{v}_{d}^{2} & \mathbf{v}_{d}^{3}\end{array}\right]^{\top}$ where $\mathbf{v}_{b}^{1}=\mathbf{y}_{b}^{1}, \mathbf{v}_{b}^{2}=$ $\mathbf{y}_{b}^{1 \times} \mathbf{y}_{b}^{2} /\left|\mathbf{y}_{b}^{1 \times} \mathbf{y}_{b}^{2}\right|, \mathbf{v}_{b}^{3}=\mathbf{v}_{b}^{1 \times} \mathbf{v}_{b}^{2}$ and $\mathbf{v}_{d}^{1}=\mathbf{y}_{d}^{1}, \mathbf{v}_{d}^{2}=\mathbf{y}_{d}^{1 \times} \mathbf{y}_{d}^{2} /\left|\mathbf{y}_{d}^{1 \times} \mathbf{y}_{d}^{2}\right|$ and $\mathbf{v}_{d}^{3}=\mathbf{v}_{d}^{1 \times} \mathbf{v}_{d}^{2}$. In terms of the Euler angle, $\phi$, and Euler axis, $\mathbf{a}$, the initial spacecraft attitude is $\phi(0)=45^{\circ}, \mathbf{a}(0)=\mathbf{a}^{\prime}(0) /\left\|\mathbf{a}^{\prime}(0)\right\|$ where $\mathbf{a}^{\prime}(0)=\left[\begin{array}{lll}2 & 1 & -3\end{array}\right]^{\top}$. The initial spacecraft angular velocity is $\omega(0)=\left[\begin{array}{lll}0.01 & -0.03 & 0.015\end{array}\right]^{\top}(\mathrm{rad} / \mathrm{s})$. The initial controller states are zero. The proportional control gain is set to $k=0.0075(\mathrm{~N} \cdot \mathrm{m})$ and $\bar{u}_{i}=0.0024(\mathrm{~N} \cdot \mathrm{m})$ which together satisfy $0.0075+0.0024<0.01$.

First, a controller designed using the method presented in Section 6.1 will be used to control the spacecraft. The weights used to synthesise the SPR controller are $\mathbf{Q}_{\mathrm{LQR}}=$ $\operatorname{diag}\{1,1,1,10,10,10\}$ and $\mathbf{R}_{\mathrm{LQR}}=\operatorname{diag}\{0.15,0.15,0.15\}$, while $\mathbf{Q}_{\mathrm{c}}=\operatorname{diag}\{150,150,150,150,150,150\}$. In Fig. $2 a$ is the attitude error in terms of the Euler angle $\phi_{e}$, and the (Euclidian) norm of the angular velocity, both against orbit. Clearly, the spacecraft attitude error goes to zero. In Fig. $2 b$ is the control torque in each axis, as well as the value of $\beta_{i}(\cdot)$, against orbit. Notice that $\beta_{i}\left(u_{i}\right) \neq 1$ between $t=0$ orbits and $t=0.05$ orbits indicating that saturation is actively being prevented. Without the presence of $\sigma(\cdot)$ and $\boldsymbol{\beta}(\cdot)$, the desired angular velocity control command plus the desired proportional control command could saturate the actuators.

Next, closed-loop control using the filtered PD controller presented in Section 6.2 will be compared to closed-loop control using the PD controller in (16). The same initial conditions, noise, $k$, and $\bar{u}_{i}$ previously stated are used once again. The value of $k_{d}$ is initially chosen using (20)-(22) given $k, I_{2}$ and $r_{e}=1.05 \times 10^{-5}\left(\mathrm{rad}^{2}\right)$ and $r_{\omega}=1.03 \times 10^{-7}\left(\mathrm{~s}^{-2}\right)$, and then further tuned during simulation leading to $k_{d}=(\mathrm{N} \cdot \mathrm{m} \cdot \mathrm{s})$. Next, $T$ is chosen so that $g_{\mathrm{c}}(s)=$ $k_{d} /(T s+1)$ rolls-off below the lowest frequency component of the angular velocity noise, and then further tuned using (20)-(22), (25), (26) and (28), leading to $T=0.5(\mathrm{~s})$. The closed-loop system response as controlled by both the PD and the filtered PD controllers is shown in Fig. 3. Notice that the overall attitude and angular velocity response is almost identical indicating that using the filtered PD controller is not necessarily accompanied by a significant performance penalty. However, of note is norm of $\boldsymbol{\tau}_{\mathrm{c}}$. Consider the norm of $\boldsymbol{\tau}_{\mathrm{c}}$ when the system has essentially reached steady state. Between $t_{1}=0.15$ orbits and $t_{2}=0.2$ orbits $\left\|\boldsymbol{\tau}_{\mathrm{c}}\right\|_{\left[t_{1}, t_{2}\right]}=1.0305 \times 10^{-3}(\mathrm{~N} \cdot \mathrm{m})$ when the filtered PD controller is used, while $\left\|\boldsymbol{\tau}_{\mathrm{c}}\right\|_{\left[t_{1}, t_{2}\right]}=2.3073 \times 10^{-3}(\mathrm{~N} \cdot \mathrm{m})$ when constantgain derivative control is used. Over this time window the norm of $\boldsymbol{\tau}_{\mathrm{c}}$ is 2.2389 times greater when constant-gain PD control is used compared to when filtered PD controller is used. This is because the SPR controller filters out the noise in the angular velocity measurement. As such, when noise corrupts the angular velocity measurement, which is arguably always the case, using the method proposed in this paper is clearly advantageous compared to using constant-gain derivative control.

Now closed-loop control using the saturation prevention technique presented in [10] will be compared to the closed-loop control using the filtered PD controller presented in Section 6.2. In [10], the control law

$$
\boldsymbol{\tau}_{\mathrm{c}}=-k\left[\sum_{i=1}^{3} a_{i}\left(\mathbf{E} \mathbf{1}_{i}\right)^{\times} \mathbf{1}_{i}\right]-k_{d, \omega}(\boldsymbol{\omega}) \boldsymbol{\omega}
$$

is presented where

$$
\begin{aligned}
k & =\frac{\alpha_{1}}{a_{1}+a_{2}+a_{3}}, \\
k_{d, \omega}(\omega) & =\alpha_{2} \operatorname{diag}\left\{\frac{1}{1+\left|\omega_{1}\right|}, \frac{1}{1+\left|\omega_{2}\right|}, \frac{1}{1+\left|\omega_{3}\right|}\right\}
\end{aligned}
$$

$0<a_{1}<a_{2}<a_{3}<\infty$, and $\mathbf{1}_{1}=\left[\begin{array}{lll}1 & 0 & 0\end{array}\right]^{\top}, \mathbf{1}_{2}=\left[\begin{array}{lll}0 & 1 & 0\end{array}\right]^{\top}$ and $\mathbf{1}_{3}=\left[\begin{array}{lll}0 & 0 & 1\end{array}\right]^{\top}$. A similar control law to that of (29) is presented in [11]. A nice feature of the controller in (29), and the controller in [11], is that the $a_{1}, a_{2}$ and $a_{3}$ terms can be used in addition to $\alpha_{1}$ and $\alpha_{2}$ as 'tuning knobs' to further tune the closedloop response about individual axes. However, (9) and (16) also have some desirable features that (29) does not. It is shown in [10] that $\left|\tau_{\mathrm{c}, i}\right| \leq \alpha_{1}+\alpha_{2}$, and hence actuator saturation is prevented if $\alpha_{1}+\alpha_{2}<\bar{\tau}_{\mathrm{c}, i}$. Recall that (9) and (16) guarantee that $k+\bar{u}_{i}<\bar{\tau}_{\mathrm{c}, i}$ thereby preventing actuator saturation. Comparing $\alpha_{1}+\alpha_{2}<\bar{\tau}_{\mathrm{c}, i}$ associated with (29) and $k+\bar{u}_{i}<\bar{\tau}_{\mathrm{c}, i}$ associated with (9) and (16), the maximum torque in each axis is the same when (29) is used for closed-loop control, while the maximum torque in each axis is 

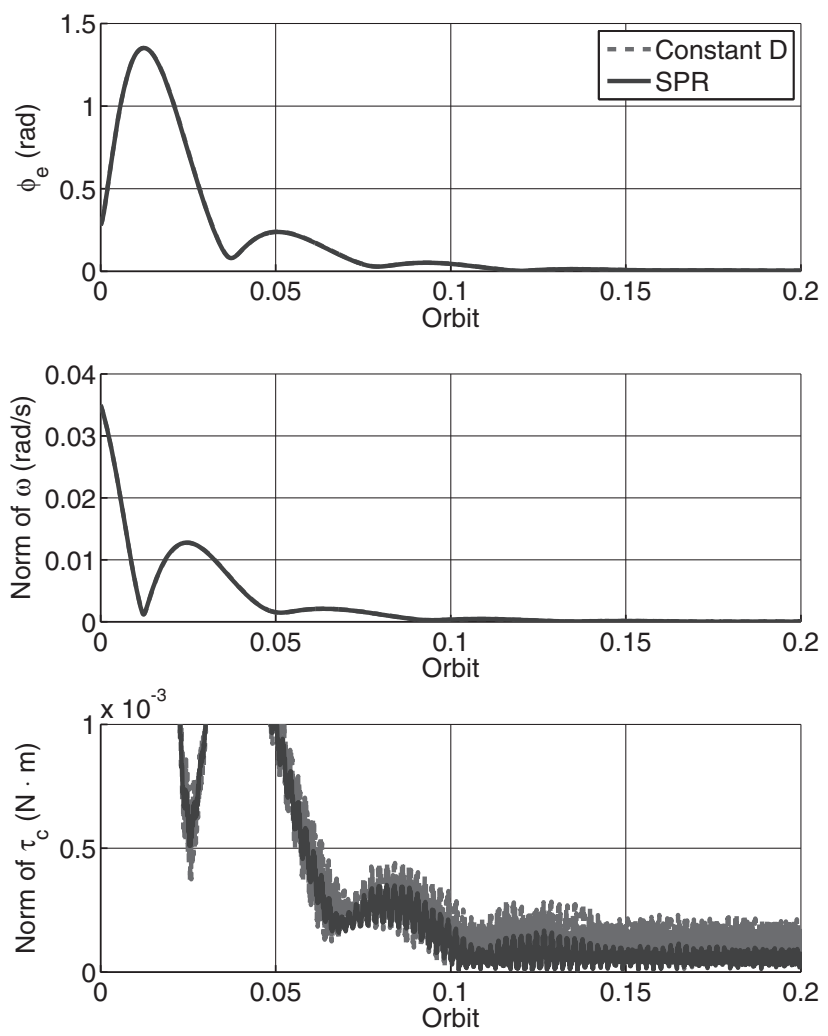

Fig. 3 Closed-loop system response subject to noisy measurements; constant-gain derivative control and filtered PD (SPR) control

permitted to be different when (9) or (16) is employed for closedloop control. This design freedom in (9) and (16) permits the use of different sized actuators, or if a particular actuator is exhibiting faulty behaviour, the overall torque applied by that actuator can be limited without affecting the remaining actuators. The control law (29) does not permit such tailoring. Moreover, the angular velocity gain in (9) and (16) is decoupled from the angular velocity saturation limit, $\bar{u}_{i}$, unlike the control law (29) where the angular velocity gain is effectively the angular velocity saturation limit. Again, (9) and (16) afford more design freedom. In addition, like the control laws (9) and (16) (see the discussion at the end of Section 4), (29) is conservative in that the proportional control component and the angular velocity control component have know knowledge of each other; if the proportional control in one axis is not saturating then the angular velocity control in the same axis does not have to be limited in such a conservative fashion. Next, notice that the angular velocity control in (29) is

$$
\boldsymbol{\tau}_{d, r}=-\alpha_{2}\left[\begin{array}{lll}
\frac{\omega_{1}}{1+\left|\omega_{1}\right|} & \frac{\omega_{2}}{1+\left|\omega_{2}\right|} & \frac{\omega_{3}}{1+\left|\omega_{3}\right|}
\end{array}\right]^{\top}
$$

The effective angular velocity control is reduced for any value of $\omega_{i}, i=1,2,3$. As such, this angular velocity control will be referred to reduced $D$ control. In contrast, in (9) and (16), for $i=1,2,3$ the $\sigma_{i}(\cdot)$ and $\beta_{i}(\cdot)$ functions are dormant unless an individual $u_{i}$ exceeds $\bar{u}_{i}$. If a $u_{i}$, which is a function of $\omega_{i}$ via (10), is below $\bar{u}_{i}$, the applied angular velocity control is not modified. The control given in (9), where the SPR controller in (10) is synthesised using either method presented in Sections 6.1 or 6.2 , and even the constant gain PD controller given in (16), afford a designer more design freedom and does not limit the effective angular velocity control as severely as the control law given in (29). Consider now the closed-loop system response as controlled by both the controller in (29) and the filtered PD controller (i.e. control law (9) and (10) along with the synthesis method in Section 6.2). Simulation results are shown in Fig. 4. The filtered PD gains from the previous simulation are used. The gains in
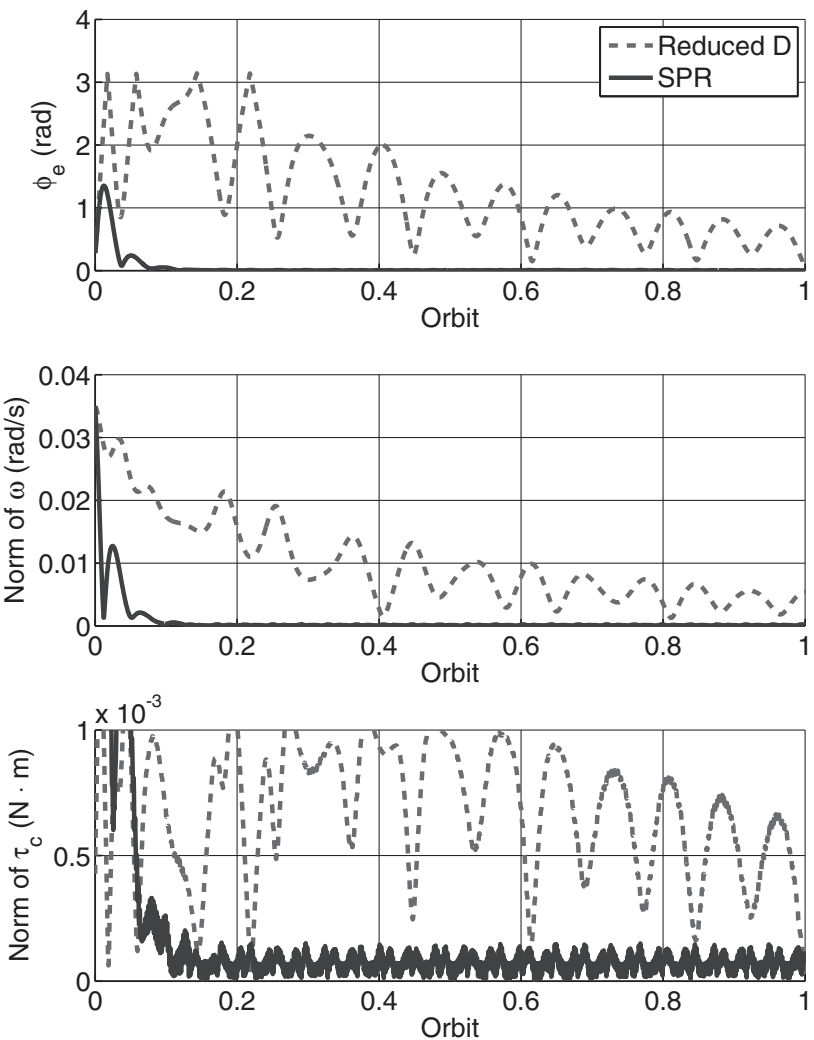

Fig. 4 Closed-loop system response subject to noisy measurements; control from the existing literature and filtered PD (SPR) control

(29) are $\alpha_{1}=0.0015(\mathrm{~N} \cdot \mathrm{m}), \alpha_{2}=0.0084(\mathrm{~N} \cdot \mathrm{m} \cdot \mathrm{s}), a_{1}=0.99$, $a_{2}=1$ and $a_{3}=1.01$. The values of $\alpha_{1}$ and $\alpha_{2}$ ensure torques in each axis are strictly less than $0.01(\mathrm{~N} \cdot \mathrm{m})$. If $\alpha_{1}=0.0075(\mathrm{~N} \cdot \mathrm{m})$ and $\alpha_{2}=0.0024(\mathrm{~N} \cdot \mathrm{m} \cdot \mathrm{s})$ are used the response degrades further. The reason the closed-loop response as controlled by the filtered PD law is superior to the closed-loop response controlled via (29) is that the angular velocity control in (29) is reduce for any non-zero value of $\omega$. The filtered PD law does not limit the angular velocity unless saturation must actively be prevented. However, the most important point is that (29) does not actively filter out noise in the angular velocity measurement. On the other hand, the filtered PD law employs a simple filter that allows high-frequency noise to be filtered.

Last, the impact of changing $k$ while keeping the filtered PD controller constant will be investigated. Specifically, the proportional control gain $k$ will be varied between 0.0038 and $0.0098(\mathrm{~N} \cdot \mathrm{m})$ at increments of $0.002(\mathrm{~N} \cdot \mathrm{m})$. To prevent actuator saturation $\bar{u}_{i}$ is set equal to $\bar{u}_{i}=k-0.01-\delta$, where $\delta=$ $0.0001(\mathrm{~N} \cdot \mathrm{m})$ for each value of $k$. The spacecraft properties, initial conditions, noise and so on, used in simulation remain unchanged from the previous simulations presented. Each value of $k$ satisfies (12). Closed-loop simulation results are presented in Fig. 5. The closed-loop response when $k=0.0075(\mathrm{~N} \cdot \mathrm{m})$ is included in Fig. 6 also. For low values of $k$ the system response is overdamped, and for high values of $k$ the system response is underdamped. Plotted in Fig. 5 is $\phi_{e},\|\boldsymbol{\omega}\|$ and $\left\|\boldsymbol{\tau}_{\mathrm{c}}\right\|$ against $k$. The attitude error, $\phi_{e}$, against $k$ curve has a minimum at $k=0.0075(\mathrm{~N} \cdot \mathrm{m})$; for low and high values of $k$ the attitude error $\phi_{e}$ increases compared to when $k=0.0075(\mathrm{~N} \cdot \mathrm{m})$. The $\|\omega\|$ and $\left\|\boldsymbol{\tau}_{\mathrm{c}}\right\|$ against $k$ curves increase modestly as between $k=0.0038$ and $0.008(\mathrm{~N} \cdot \mathrm{m})$. Above $k=0.008(\mathrm{~N} \cdot \mathrm{m})$ the $\phi_{e},\|\omega\|$ and $\left\|\boldsymbol{\tau}_{\mathrm{c}}\right\|$ against $k$ curves increase sharply indicating lager attitude and angular velocity errors as well as in increased control effort. In order to reduce attitude error, angular velocity error, and the control effort, $k$ should be kept small. Unfortunately, via (12), the set of allowable initial conditions is reduced when $k$ is reduced. 

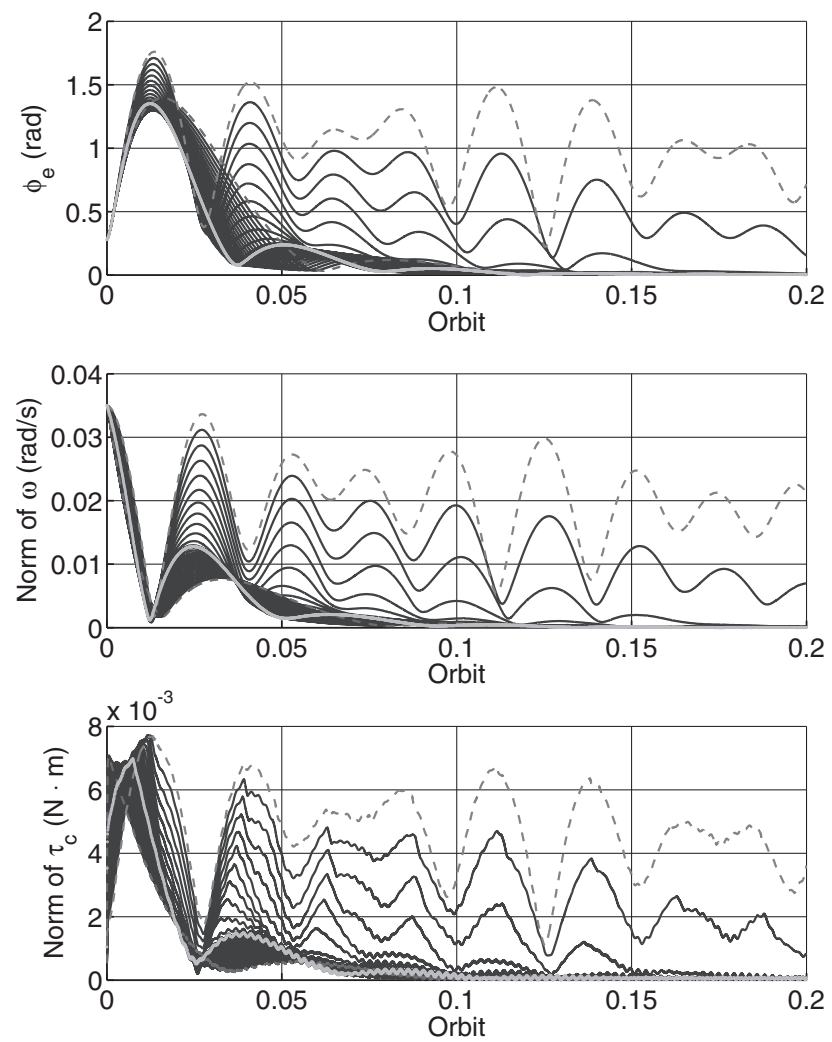

Fig. 5 Closed-loop system response subject to noisy measurements while varying $k$ between 0.0038 and $0.0098(\mathrm{~N} \cdot \mathrm{m})$

The two dashed lines are associated with $k_{p}=0.0038(\mathrm{~N} \cdot \mathrm{m})$ and $k_{p}=0.0098(\mathrm{~N} \cdot \mathrm{m})$, while the light solid line is associated with $k_{p}=$ $0.0075(\mathrm{~N} \cdot \mathrm{m})$
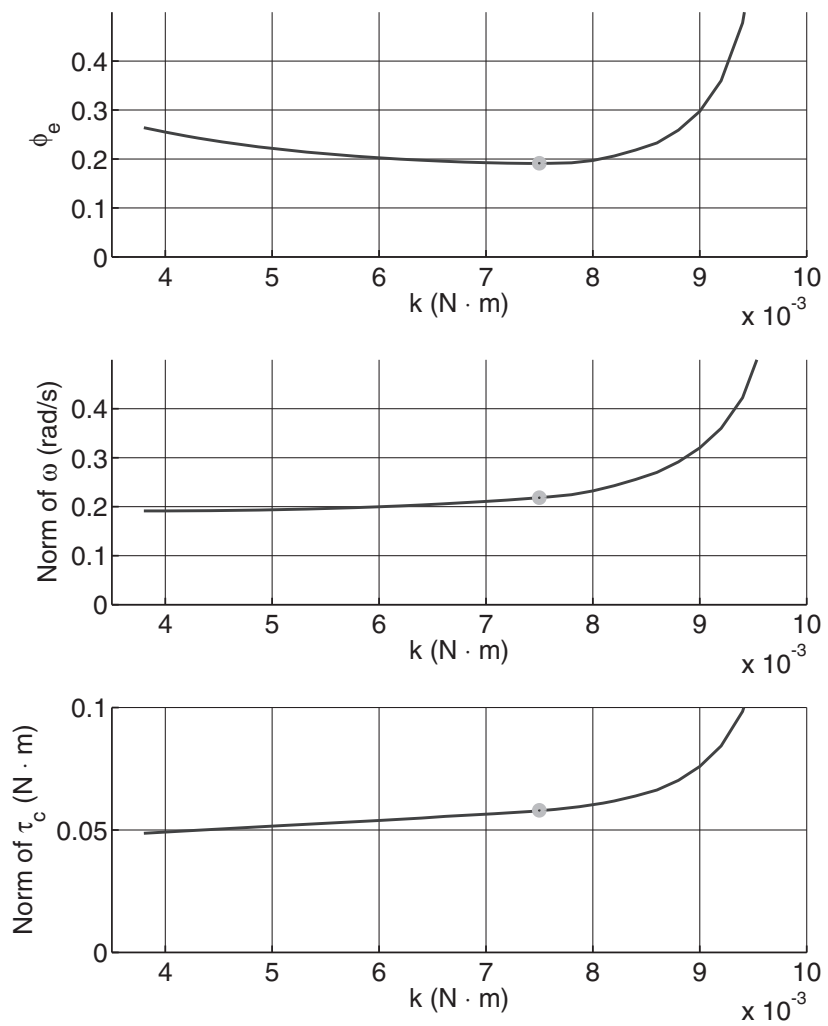

Fig. 6 Norm of $\phi_{e}, \omega$ and $\boldsymbol{\tau}_{\mathrm{c}}$ against $k$

The dots correspond to $k=0.0075(\mathrm{~N} \cdot \mathrm{m})$. Notice in the norm of $\phi_{e}$ against $k$ plot the dot is at the curves inflection point

\section{Closing remarks}

In this paper, set-point attitude control of a rigid body explicitly disallowing actuator saturation is considered. The particular form of the control method ensures that actuator saturation is prevented and asymptotic stability of a desired closed-loop equilibrium point is guaranteed. The control architecture is composed of a proportional control term, and an angular velocity control term. The proportional control term is a function of the attitude error as described by the attitude error DCM, and is naturally bounded. The angular velocity control term is also restricted by a saturation function. The angular velocity control is generated by an SPR system driven by an input non-linearity. The form of the SPR system's input non-linearity is dictated by the saturation function. Two SPR synthesis methods are considered. The first considered designing the SPR system matrices using the LQR formulation and the KYP lemma. The second considered the use of a simple low-pass filter that is naturally SPR thereby realising a filtered PD controller. This is significant because in practice a simple low-pass filter is often used to reject high-frequency noise, while the proposed method guarantees closed-loop stability and the prevention of actuator saturation. Future work will focus on DCM-based attitude control laws that disallow actuator rate saturation, as well as magnitude saturation. In addition, an interesting avenue to explore is the use of non-linear antiwindup techniques, such as [32], for control without parameterising the DCM.

\section{References}

1 Wie, B., Weiss, H., Arapostathis, A.: 'Quaternion feedback regulator for spacecraft eigenaxis rotations', AIAA J. Guid. Control Dyn., 1989, 12, (3), pp. $375-380$

2 Slotine, J.-E., Di Benedetto, M.: 'Hamiltonian adaptive control of spacecraft', IEEE Trans. Autom. Control, 1990, 35, pp. 848-852

3 Wen, J.T.-Y., Kreutz-Delgado, K.: 'The attitude control problem', IEEE Trans. Autom. Control, 1991, 36, (10), pp. 1148-1162

4 Egeland, O., Godhavn, J.-M.: 'Passivity based adaptive attitude control of a rigid spacecraft', IEEE Trans. Autom. Control, 1994, 39, (4), pp. 842-846

5 Joshi, S.M., Kelkar, A.G., Wen, J.T.-Y.: 'Robust attitude stabilization of spacecraft using nonlinear quaternion feedback', IEEE Trans. Autom. Control, 1995, 40, (10), pp. 1800-1803

6 Tayebi, A.: 'Unit quaternion-based output feedback for the attitude tracking problem', IEEE Trans. Autom. Control, 2008, 53, (6), pp. 1516-1520

7 Chaturvedi, N.A., Sanyal, A.K., McClamroch, N.H.: 'Rigid-body attitude control', IEEE Control Syst. Mag., 2011, 31, (3), pp. 30-51

8 Bhat, S.P., Bernstein, D.S.: 'A topological obstruction to continuous global stabilization of rotational motion and the unwinding phenomenon', Syst. Control Lett., 2000, 39, pp. 63-70

9 Bullo, F., Murray, R.M.: 'Tracking for fully actuated mechanical systems: a geometric framework', Automatica, 1999, 35, pp. 17-34

10 Sanyal, A., Fosbury, A., Chaturvedi, N., Bernstein, D.S.: 'Inertia-free spacecraft attitude tracking with disturbance rejection and almost global stabilization', AIAA J. Guid. Control Dyn., 2009, 32, (4), pp. 1167-1178 doi: 10.2514/1.41565.

11 Chaturvedi, N.A., McClamroch, N.H.: 'Almost global attitude stabilization of an orbiting satellite including gravity gradient and control saturation effects'. Proc. of the American Control Conf., Minneapolis, MN, USA, 14-16 June, 2006, pp. $1748-1753$

12 Khosravian, A., Namvar, M.: 'Rigid body attitude control using a single vector measurement and gyro', IEEE Trans. Autom. Control, 2012, 57, pp. 1273-1279

13 Lee, T.: 'Exponential stability of an attitude tracking control system on $\mathrm{SO}(3)$ for large-angle rotation maneuvers', Syst. Control Lett., 2012, 61, pp. 231-237

14 Lee, T.: 'Robust adaptive attitude tracking on $\mathrm{SO}(3)$ with and application to a quadrotor UAV', IEEE Trans. Control Syst. Technol., 2013, 21, (5), pp. $1924-1930$

15 Mahony, R., Hamel, T., Pflimlin, J.-M.: 'Nonlinear complementary filters on the special orthogonal group', IEEE Trans. Autom. Control, 2008, 53, (5), pp. $1203-1218$

16 Mahony, R., Euston, M., Kim, J., Coote, P., Hamel, T. 'A non-linear observer for attitude estimation of a fixed-wing unmanned aerial vehicle without GPS measurements', Trans. Inst. Meas. Control, 2011, 33, (6), pp. 699-717

17 Grip, H.F., Fossen, T.I., Johansen, T.A., Saberi, A.: 'Attitude estimation using biased gyro and vector measurements with time-varying reference vectors', IEEE Trans. Autom. Control, 2012, 57, (5), pp. 1332-1338

18 Tran, N.K., He, X., Zlotnik, D.E., Forbes, J.R.: 'Attitude sensing and control of a Stratospheric Balloon platform'. AIAA Balloon Systems Conf., Daytona Beach, FL, March 25-28, 2013

19 Akella, M.R., Valdivia, A., Kotamraju, G.R.: 'Velocity-free attitude controllers subject to actuator magnitude and rate saturations', AIAA J. Guid. Control Dyn., 2005, 28, (4), pp. 659-666

20 Wallsgrove, R.J., Akella, M.R.: 'Globally stabilizing saturated attitude control in the presence of bounded unknown disturbances', AIAA J. Guid. Control Dyn. 2005, 28, (5), pp. 957-963 
21 Boskovic, J.D., Li, S.-M., Mehra, R.K.: 'Robust adaptive variable structure control of spacecraft under control input saturation', AIAA J. Guid. Control Dyn., 2001, 24, (1), pp. 14-22

22 de Ruiter, A.H.J. : 'Adaptive spacecraft attitude tracking control with actuator saturation', AIAA J. Guid. Control Dyn., 2010, 33, (5), pp. 16921695

23 Bernstein, D.S., Haddad, W.M.: 'Nonlinear controllers for positive real systems with arbitrary input nonlinearities', IEEE Trans. Autom. Control, 1994, 39, (7), pp. $1513-1517$

24 Haddad, W.M., Chellaboina, V.: 'Nonlinear control of Hammerstein systems with passive nonlinear dynamics', IEEE Trans. Autom. Control, 2001, 46, (10), pp. 1630-1634

25 Benhabib, R.J., Iwens, R.P., Jackson, R.L.: 'Stability of large space structure control systems using positivity concepts', AIAA J. Guid. Control Dyn., 1981, 4, (5), pp. $487-494$
Bullo, F., Lewis, A.D.: 'Geometric control of mechanical systems' (Springer Science + Business Media, Inc., New York, 2005)

27 Murry, R.N., Li, Z., Sastry, S.S.: 'A mathematical introduction to Robotic manipulation' (CRC Press, Inc., Boca Raton, FL, 1993)

28 Hughes, P.C.: 'Spacecraft attitude dynamics' (Dover, Mineola, New York, 2nd edn., 2004)

29 Wen, J.T.: 'Time domain and frequency domain conditions for strict positive realness', IEEE Trans. Autom. Control, 1988, 33, pp. 988-992

30 Salcudean, S.: 'A globally convergent angular velocity observer for rigid body motion', IEEE Trans. Autom. Control, 1991, 36, pp. 1493-1497

31 Schuster, M.D., Oh, S.D.: 'Three-axis attitude determination from vector observations', AIAA J. Guid. Control, 1981, 4, (1), pp. 70-77

32 Morabito, F., Teel, A.R., Zaccarian, L.: 'Nonlinear antiwindup applied to Euler-Lagrange systems', IEEE Trans. Autom. Control, 2004, 20, (3), pp. $526-537$ 